\title{
Recurrent Lower Urinary Tract Infections Have a Detrimental Effect on Patient Quality of Life: a Prospective, Observational Study
}

\author{
Julien Renard · Stefania Ballarini - Teresa Mascarenhas • Mohamed Zahran • \\ Enrique Quimper · Jacques Choucair • Christophe E. Iselin
}

To view enhanced content go to www.infectiousdiseases-open.com Received: November 6, 2014 / Published online: December 18, 2014

(c) The Author(s) 2014. This article is published with open access at Springerlink.com

\section{ABSTRACT}

Introduction: This study investigated the effects of recurrent urinary tract infections (rUTI) and the impact of prophylaxis on rUTI and patients' quality of life (QoL).

Methods: Altogether, 575 patients affected by rUTI were included in a 6-month observational study. QoL was assessed using the Hospital Anxiety and Depression (HAD) and the

Electronic supplementary material The online version of this article (doi:10.1007/s40121-014-0054-6) contains supplementary material, which is available to authorized users.

J. Renard · C. E. Iselin ( $₫)$

University Hospital of Geneva, Geneva, Switzerland

e-mail: julien.renard@hcuge.ch

S. Ballarini

OM Pharma/Vifor Pharma, Geneva, Switzerland

T. Mascarenhas

Hospital de São João, Porto, Portugal

M. Zahran

Cairo University, Cairo, Egypt

E. Quimper

Universidad Nacional Mayor de San Marcos,

Lima, Peru

J. Choucair

Hotel Dieu de France, Saint Joseph University,

Beirut, Lebanon
Leicester scales. Statistical analyses were performed using SAS $^{\circledR}$ Version 8.2 software (SAS Institute Inc., Cary, NC, USA). The significance level was set at $5 \%$. Spearman correlation was used to assess the degree of correlation between infectious episodes and HAD and Leicester scores. For each parameter, the comparison between Day 0 and Day 180 was performed using Wilcoxon signed-rank test for quantitative data.

Results: In total, $61.9 \%$ of patients suffering from rUTI exhibited some degree of depression at baseline (Day 0). Alternative oral nonantimicrobial prophylactic treatment for rUTI [Escherichia coli lyophilized bacterial lysate (OM89)] was administered to $94.4 \%$ of patients (1 capsule a day for 90 days), followed by a 3-month treatment-free period. At the end of the study (Day 180), the mean number of UTI decreased by $59.3 \%(P \leq 0.0001)$, the total HAD score decreased by $32.1 \%(P \leq 0.0001)$, and the mean Leicester score decreased by $44.0 \%$ $(P \leq 0.0001)$ from baseline. There was a correlation trend between the reduction in the numbers of UTI at the end of the study compared to the 6 months prior to study entry and the reduction in the anxiety, depression, 
total HAD scores, activity, feeling, and total Leicester scores registered from Day 0 to Day 180, suggesting a lessening of emotional problems, and social and functional handicaps with decreasing UTI incidence.

Conclusions: This study showed that rUTI had a negative impact on patients' QoL and that effective alternative prophylaxis significantly improved their QoL.

Keywords: Cystitis; Prophylaxis; Quality of life; Urinary tract infection

\section{INTRODUCTION}

Acute lower urinary tract infections (UTIs) in adults consist of episodes of acute cystitis. They occur in otherwise healthy individuals, mostly women, with no risk factors such as underlying disease or urinary tract abnormalities [1]. It is estimated that 1 in 2 women will have at least 1 episode of UTI in their lifetime, and UTIs are at least 50 times more common in premenopausal women than in men [2]. Furthermore, up to $20 \%$ of women with acute cystitis develop recurrent uncomplicated UTIs (3 or more episodes per year) [1]. Patients experience a psychological burden because they live with the anxiety of sudden acute episodes. In the United States, UTIs are responsible for seven million medical visits a year, including two million for cystitis alone, with three million patients treated with antibiotics. The total estimated cost is $\$ 3.5$ billion; in Europe, the estimated cost is $€ 1.5$ billion $[3,4]$.

The standard symptoms of acute uncomplicated UTIs (cystitis) include acute dysuria, pollakiuria, pain and burning associated with micturition, urgent need to urinate, as well as suprapubic tenderness without fever. Escherichia coli strains are the most common uropathogens, found in $80 \%$ of urine specimens from patients with lower acute uncomplicated UTI (cystitis), followed by Staphylococcus saprophyticus (5-15\%), and aerobic Gram-negative bacteria, such as Klebsiella pneumoniae and Proteus mirabilis (5-10\%) [5]. Although pathogen identification is essential for testing antimicrobial susceptibility and selecting appropriate therapy, it is not routinely performed. In the European Association of Urology (EAU) guidelines [1], pathogen identification is recommended only in cases of pregnancy, recurring UTI, or fever and flank pain (to exclude acute pyelonephritis) [6].

Empirical courses of antibiotics are generally effective in treating cystitis (single dosing or courses of 3-7 days), and the recommended antibiotics include fosfomycin, nitrofurantoin, cephalosporins, and trimethoprimsulfamethoxazole (TMP-SMX) [7]. However, bacterial re-colonization can lead to relapses or re-infections due to bacterial reservoirs [8] or drug resistance [9]. Alternative effective approaches to managing recurrent uncomplicated UTIs comprise risk factor evaluation, patient counseling, behavioral modification, and non-antimicrobial prophylaxis. Several non-antimicrobial prophylactic agents are recommended by the current EAU guidelines including probiotics and oral immunostimulation with bacterial extracts of $E$. coli (OM-89) [1]. Oral immunostimulation with OM-89 was given a Grade B recommendation in the guidelines due to its well-documented efficacy and good tolerability profile (and was rated as having the highest level of clinical evidence, 1a). The advantage of non-antimicrobial prophylaxis is that, as compared to antibiotics, it that does not contribute to the growing problem of bacterial antibiotic resistance. This is particularly 
relevant for the treatment of UTIs due to the paucity of antibiotics effective against Gramnegative bacteria. OM-89 has been shown to enhance the immune defenses against recurrent UTIs (rUTIs) [10], and is a valuable component in UTI treatment. Several well-designed doubleblind placebo-controlled studies and two published meta-analyses involving over 1,000 patients [11-15] have shown that, when administered over a 3-month period, OM-89 decreased the risk of recurrence, and significantly reduced the signs and symptoms of UTI, as well as the need for antibiotics over a period of 12 months. However, these studies have not addressed the effects of rUTIs on quality of life (QoL).

Despite the social and economic relevance, to date, few publications have focused on the impact of frequent UTIs on QoL. Treating repeated acute infections alone is not enough to improve the QoL of these patients. Determining the patient QoL is a key element in assessing treatment efficacy, a consideration that is at times neglected by health care professionals. The sudden, unforeseeable, and distressing nature of painful UTI episodes often causes patient anxiety. The resulting social handicap is known to induce feelings of selfdevaluation or culpability, which can lead to clinical symptoms of depression [16-20]. This social handicap particularly affects premenopausal working women who constitute a significant proportion of the population experiencing UTIs. Furthermore, rUTIs can result in functional handicap, particularly in elderly patients in whom infectious episodes may provoke or aggravate incontinence, leading to rapid deterioration of general health or initiating confusion [21-24]. All of these issues may significantly impact patient QoL.
Previous small-scale studies [18, 25] and observations from clinical practice have indicated that recurrent cystitis has a negative impact on QoL. Consequently, this observational pilot study aimed to substantiate the notion that recurrent cystitis negatively impacts QoL. To this end, the impact of rUTIs on patient QoL was evaluated, and changes in patient QoL during preventative treatment (prescribed by the attending physician) were sought for.

\section{METHODS}

\section{Study Design and Population}

This international, prospective, observational, multi-center, epidemiological pilot study was conducted during 2005-2006, in accordance with the ethical standards of the responsible committees on human experimentation (institutional and national) and with the 1975 Declaration of Helsinki, as revised in 2000 and 2008. Due to limited evidence of the impact of rUTIs on QoL, this study was designed to evaluate whether rUTIs have a negative impact on patient QoL. The study did not interfere with usual patient management, no special treatment or examination was stipulated, and the physician's prescribing freedom was maintained. The study population consisted of patients affected by lower rUTIs (defined as $\geq 3$ infectious episodes over the previous 12 months) who were treated by urologists, gynecologists, hospital doctors, or general practitioners. Patients were male or female, $>18$ years of age, suffering from lower rUTIs, and with a planned preventative treatment. Patients unable to understand or complete the QoL questionnaires, unwilling to participate, who had a deformity of the urinary tract, 
neurogenic bladder, or presenting with symptoms of vaginitis were excluded from the study.

Data were collected from 151 centers in seven countries: Egypt, Germany, Lebanon, Peru, Poland, Portugal, and Switzerland. To ensure even geographical distribution and fair representation of patients from different geographical areas, physicians were asked to recruit a maximum of five patients meeting the eligibility criteria and willing to participate in the study. Each patient was examined at inclusion (Day 0) and 6 months later (Day 180).

This observational pilot study aimed to substantiate the notion that recurrent cystitis has a negative impact on QoL. The primary endpoint of the study was the relationship between the number of infectious episodes and the Hospital Anxiety and Depression (HAD) scale [26]. The secondary endpoint was the relationship between the Leicester impact scale [27] and the number of infectious episodes.

\section{Evaluation Methods}

\section{Assessments on Day 0 and Day 180}

On Day 0 and Day 180, physicians recorded the patient UTI history (date of first infection, date of start of recurrent signs, frequency of infectious episodes, triggering factors, and treatments received), description of urinary symptoms concerning the last infection, description of pain (intensity, duration, and moments of appearance), previous microbiological data (date of last examination and microbiological diagnosis), and number of UTI episodes over the previous 6 months. To assess the presence and level of emotional problems, as well as the social and functional handicap in relation to rUTIs, the physician asked the patient to complete the HAD scale and Leicester impact scale questionnaires.

\section{Assessment Scales}

QoL is an outcome that can only be assessed by the subjects themselves, without reference to any norm. This is a specific approach in which the patients express their perception of the situation and the satisfaction (or dissatisfaction) they experience compared with their expectations [28]. Therefore, QoL assessment was made using two specific questionnaires: a generic form on anxiety and depression disorders (i.e., the HAD scale) [26] and a UTIspecific questionnaire on the functional and social handicaps associated with UTIs (i.e., the Leicester impact scale) [27].

\section{Assessment of Emotional Problems}

Emotional problems experienced by patients during the previous week were assessed using the HAD scale, which provides a reliable method of detecting the most common signs of anxiety and depressive disorders [26]. The questionnaire comprises 14 questions (7 evaluate anxiety and 7 evaluate depression). Each reply has a value of $0-3$, and the assessment was made in a semi-quantitative manner using an adverb or qualifying short sentence to evaluate symptom intensity. Therefore, the possible total score ranged from 0 to 42 , with both anxiety and depression subscales ranging from 0 to 21 .

The HAD scores were divided into three ranges to assess the presence of anxiety or depression: $\quad 0-7=$ normal, $\quad 8-10=$ possibly indicative of anxiety or depression, and $\geq 11=$ probable presence of anxiety or depression. A score of at least 8 on either anxiety or depression subscales indicated an emotional disorder. 
Assessment of Social or Functional Handicap

Social or functional handicap was assessed using the Leicester impact scale. This is a valid and reliable, interviewer-administered QoL tool for patients with urinary storage symptoms [27]. It was used to determine the impact of urinary problems on daily activities (part 1) and on mood and behavior (part 2). Part 1 of the scale assessed nine situations in which urinary infections are likely to interfere with daily activities (activity score), categorized as often, rarely, never, activity stopped because of urinary problems, and activity stopped for other reasons. Part 2 of the scale was used to determine the impact of symptoms on mood and behavior (feeling score), categorized as often, rarely, or never. The Leicester impact scale was divided into four ranges: 0-14, 15-20, 21-30, and 31-42. A score of $>14$ indicated that the patient had some degree of functional or social handicap, a score of $>20$ was suggestive of a major social or functional handicap, and scores $>31$ indicated major social and functional handicaps.

\section{Statistical Analysis}

Statistical analyses were performed using $\mathrm{SAS}^{\circledR}$ Version 8.2 software (SAS Institute Inc., Cary, NC, USA). The significance level was set at 5\%. Spearman correlation was used, as appropriate, to assess the degree of correlation between infectious episodes and the HAD and Leicester scores. For each parameter, the comparison between Day 0 and Day 180 was performed using Wilcoxon signed-rank test for quantitative data.

\section{RESULTS}

\section{Patient Disposition}

A total of 699 patients from seven countries were initially enrolled in the study. The mean patient age was 46.3 years. Among study participants, $12.3 \%$ were male and $87.7 \%$ were female. Data of 43 patients from Portugal were accidently lost, and of 656 remaining patients 81 patients $(12.3 \%)$ were lost to follow-up. Therefore, 575 patients $(87.6 \%)$ completed the 6-month (Day 180) follow-up visit and were included in the primary endpoint analysis ('analysis population'). All patients who received OM-89 prophylaxis at the standard posology of 90 consecutive days (about 3 months) were followed up for a period of 3 months after treatment discontinuation.

\section{Baseline and Day 180 Results}

The most frequently reported urinary symptoms concerning the last infection were recorded at Day 0 (baseline). These were: pain and/or burning during micturition (93.7\%), pollakiuria (82.4\%), and dysuria (80.1\%). Approximately, one-third (29.8\%) of patients reported asthenia. On Day 180, the population of patients suffering from asthenia or dysuria was reduced by approximately half, and there was a reduction of over $30 \%$ in the number of patients suffering from pollakiuria or pain/ burning during micturition (Fig. 1).

The Wilcoxon test $(P \leq 0.0001)$ showed that the mean number of urinary infections was 2.7 [standard deviation $(\mathrm{SD})=1.2$ ] during the 6 months prior to study entry, measured on Day 0, and 1.1 $(\mathrm{SD}=1.1)$ during the 6-month study period, measured on Day 180; this represents a statistically significant decrease of $1.6(\mathrm{SD}=1.6)$.

During the study, $5 \%$ of patients did not receive any preventative treatment for UTI. Of the remaining 95\%, the vast majority (94.4\%) were given OM-89 (1 capsule a day for 90 days), followed by a 3-month treatment-free period. Among the analysis population, $53.7 \%$ of 


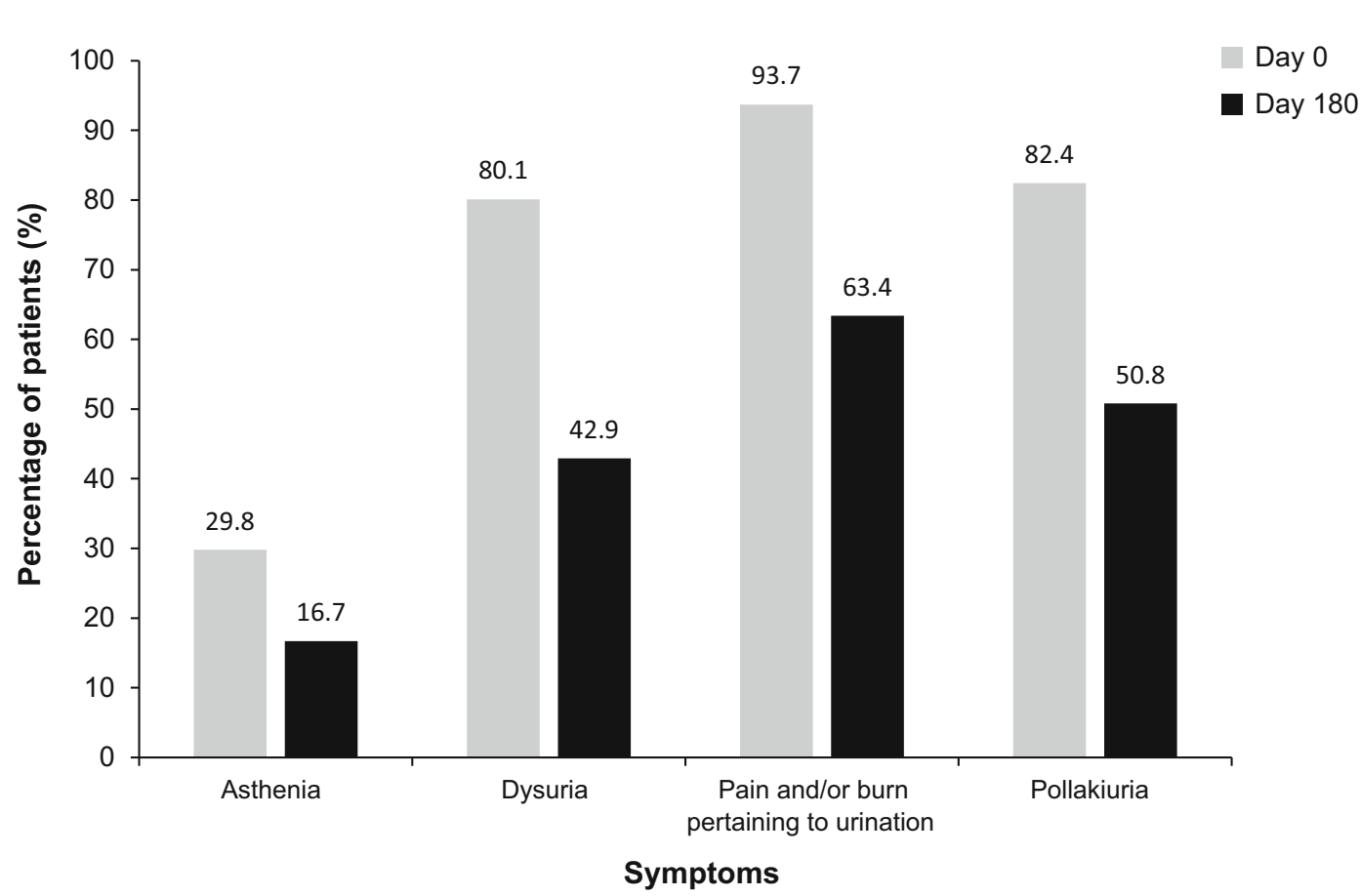

Fig. 1 Symptoms of urinary infection from the last urinary tract infection episode registered at Day 0 and Day 180analysis population $(N=575)$

patients were treated for acute UTI episodes: $41.6 \%$ of them received antibiotics, while the remaining $12.1 \%$ received unspecified other treatments.

At baseline, the HAD score revealed that 177 (32\%), $153(27.7 \%)$, and $12(2.2 \%)$ patients exhibited mild, moderate, and severe levels of depression, respectively. The mean HAD anxiety subscale score was $10.3 \quad(\mathrm{SD}=4.1$, range 0-21), and the mean HAD depression subscale score was $6.4(\mathrm{SD}=3.6$, range $0-18)$. In total, 342 patients $(61.8 \%)$ had at least minor emotional disorders, 165 (29.9\%) of whom presented with a high anxious-depressive state. At Day 180, the proportion of patients with possibly clinically relevant anxiety/depression levels (mild to severe anxiety or depression) decreased. The difference was more significant for the anxiety score, with a total reduction of $38.5 \%$ from Day 0 to Day 180 (Table 1). The total HAD score at Day 180 indicated a 30.9\% increase in patients with a normal score (below 14 ), and a total decrease of $31 \%$ in patients experiencing mild to severe anxiety or depression (total HAD scores between 15 and 42; Fig. 2). At Day 180, the mean HAD anxiety subscale score was $6.6(\mathrm{SD}=3.7)$, the mean HAD depression score was $4.8(\mathrm{SD}=3.5)$, and the total HAD score was $11.4 \quad(\mathrm{SD}=6.5)$, representing a statistically significant decrease in total HAD score of $32.1 \%$ from baseline $(P \leq 0.0001)$. The mean number of urinary episodes was $2.7(\mathrm{SD}=1.2)$ on Day 0 , and 1.1 $(\mathrm{SD}=1.1)$ on Day 180 , showing a statistically significant decrease of $59.3 \%$ from baseline $(P \leq 0.0001)$. Thus, there was a correlation trend between the reduction in the numbers of UTIs from Day 0 to Day 180 and the reduction in the anxiety, depression, and total HAD scores, suggesting a lessening of emotional problems with decreasing UTI incidence (Fig. 3). 
Table 1 HAD depression and anxiety scores at Day 0 and Day $180(N=575)$

\begin{tabular}{llllll}
\hline Range & \multicolumn{2}{l}{ Depression score, \% of patients } & & \multicolumn{2}{l}{ Anxiety score, \% of patients } \\
\cline { 2 - 3 } & Day 0 & Day 180 & & Day 0 & Day 180 \\
\hline $0-7$ (normal) & 64.4 & 77.2 & & 26.7 & 65.2 \\
$8-10$ (mild) & 22.7 & 15.4 & & 28.2 & 19.3 \\
$11-15$ (moderate) & 12.2 & 7.1 & & 31.2 & 13.9 \\
$16-21$ (severe) & 0.7 & 0.4 & & 13.9 & 1.6 \\
\hline
\end{tabular}

$H A D$ hospital anxiety and depression

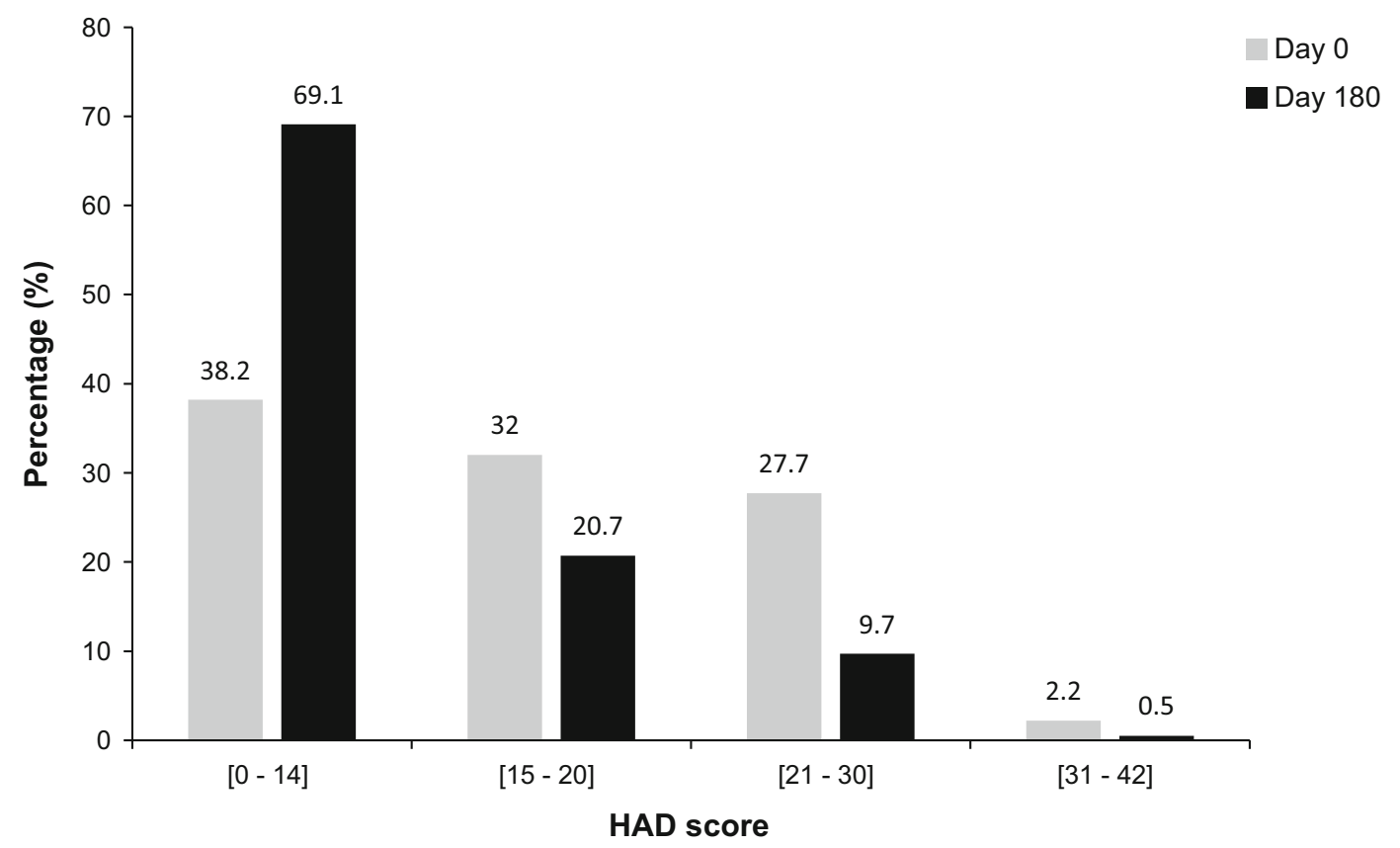

Fig. 2 Total HAD score at Day 0 and Day 180-analysis population ( $N=575$, some data is missing). The HAD questionnaire comprises 14 questions (7 evaluate anxiety and 7 evaluate depression). Each reply has a value of $0-3$,

The mean activity score was $10.4(\mathrm{SD}=4.7)$ on Day 0 , and $4.0(\mathrm{SD}=5.6)$ on Day 180 , showing a statistically significant $32.7 \%$ decrease from baseline $(P \leq 0.0001)$. The mean feeling score was $9.1(\mathrm{SD}=5.0)$ on Day 0 , and $4.1(\mathrm{SD}=4.2)$ on Day 180 , which corresponds to a statistically significant decrease of $54.9 \%$ from baseline $(P \leq 0.0001)$. The mean Leicester score decreased from 19.3 $(\mathrm{SD}=8.1)$ on Day 0 giving a possible total score ranging from 0 to 42 . Higher scores indicate higher levels of anxiety and depression. $H A D$ hospital anxiety and depression

to $10.8(\mathrm{SD}=7.9)$ on Day 180 , representing a statistically significant decrease of $44.0 \%$ compared with baseline $(P \leq 0.0001)$. The mean number of urinary episodes was 2.7 $(\mathrm{SD}=1.2)$ on Day 0 , and $1.1(\mathrm{SD}=1.1)$ on Day 180, showing a statistically significant decrease of $59.3 \%$ from baseline $(P \leq 0.0001)$. There was a correlation trend between the reduction in number of UTIs from Day 0 to 


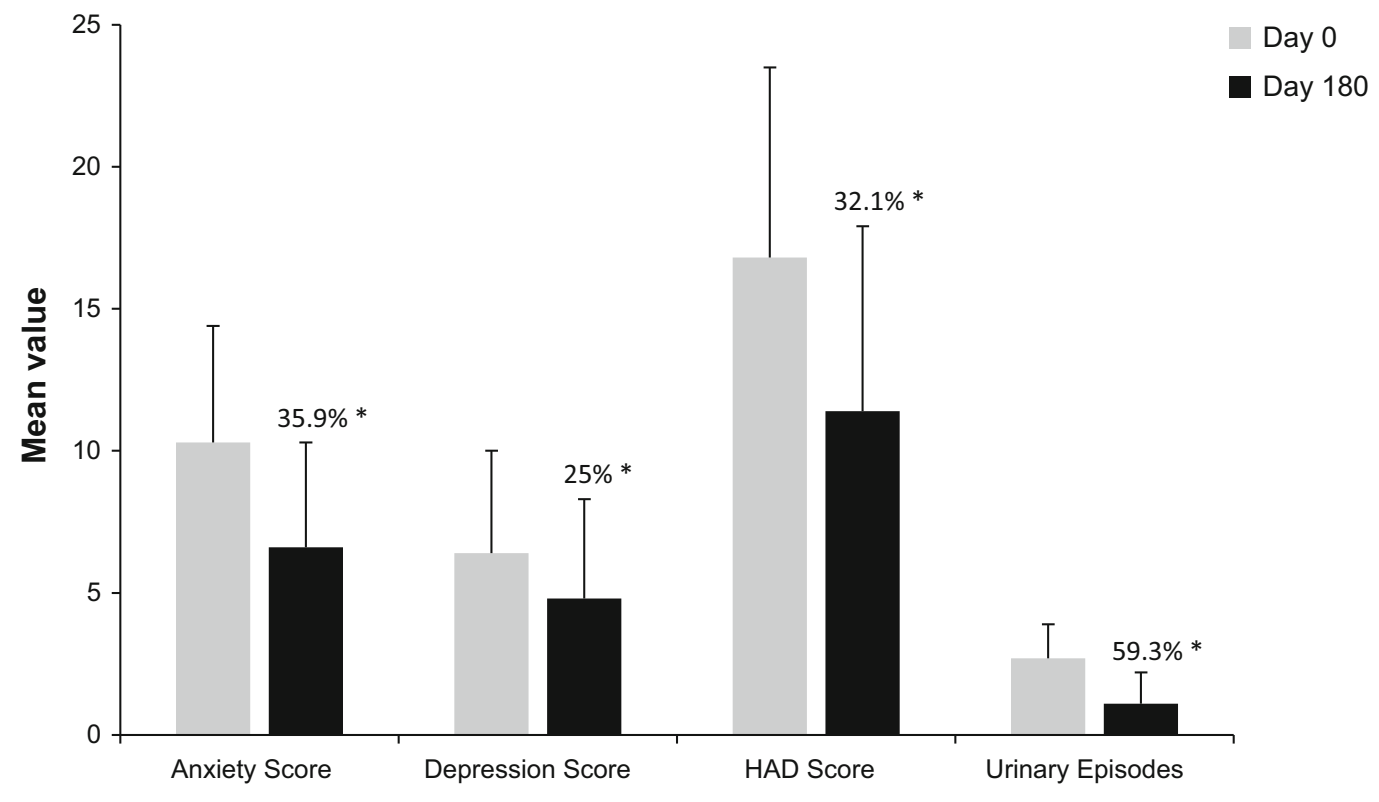

Fig. 3 Evolution of HAD subscales and global score, and number of urinary infection episodes between Day 0 and Day 180 -analysis population $(N=575)$. The HAD subscales (anxiety score and depression score) were divided into three ranges to assess the presence of anxiety or depression: $0-7$ normal, $8-10$ possibly indicative of anxiety

or depression, and $\geq 11$ probable presence of anxiety or depression. A score of at least 8 on either the anxiety subscale or depression subscale indicated an emotional disorder. Asterisk the percentage reduction from Day 0. All statistically significant $(P \leq 0.0001)$. HAD hospital anxiety and depression

Day 180 and the reduction in the activity, feeling, and total Leicester scores. These statistically significant reductions suggest a lessening of social and functional handicap with decreasing UTI incidence (Fig. 4).

Physicians reported overall improvement as 'much better' or 'very much better' in the vast majority (82.4\%) of patients. Furthermore, $12.7 \%$ of patients reported a 'slightly better' feeling, and only 3.9\% reported 'unchanged' feeling. The remaining $1 \%$ of patients did not report on their improvement.

\section{DISCUSSION}

Recurrent UTIs are a significant problem for many patients. Health professionals do not always appreciate the impact of this ostensibly minor condition on a patient's sense of well- being; successful medical treatment of each infection does not always translate into an improvement in QoL [29].

This study evaluated the impact of rUTIs on patient QoL before and during preventative treatment, and while receiving the best standard of care (i.e., during acute infection episodes). The study did not interfere with usual patient management, no special treatment or examination was stipulated, and the physician's prescribing freedom was maintained. QoL is a subjective, unempirical concept that does not have a universally accepted definition. The study used clinically validated questionnaires (HAD and Leicester scales) to assess the presence of anxiety or depression, and social or functional handicap in patients who experienced rUTIs. The questionnaires were completed by the patients themselves. Both 


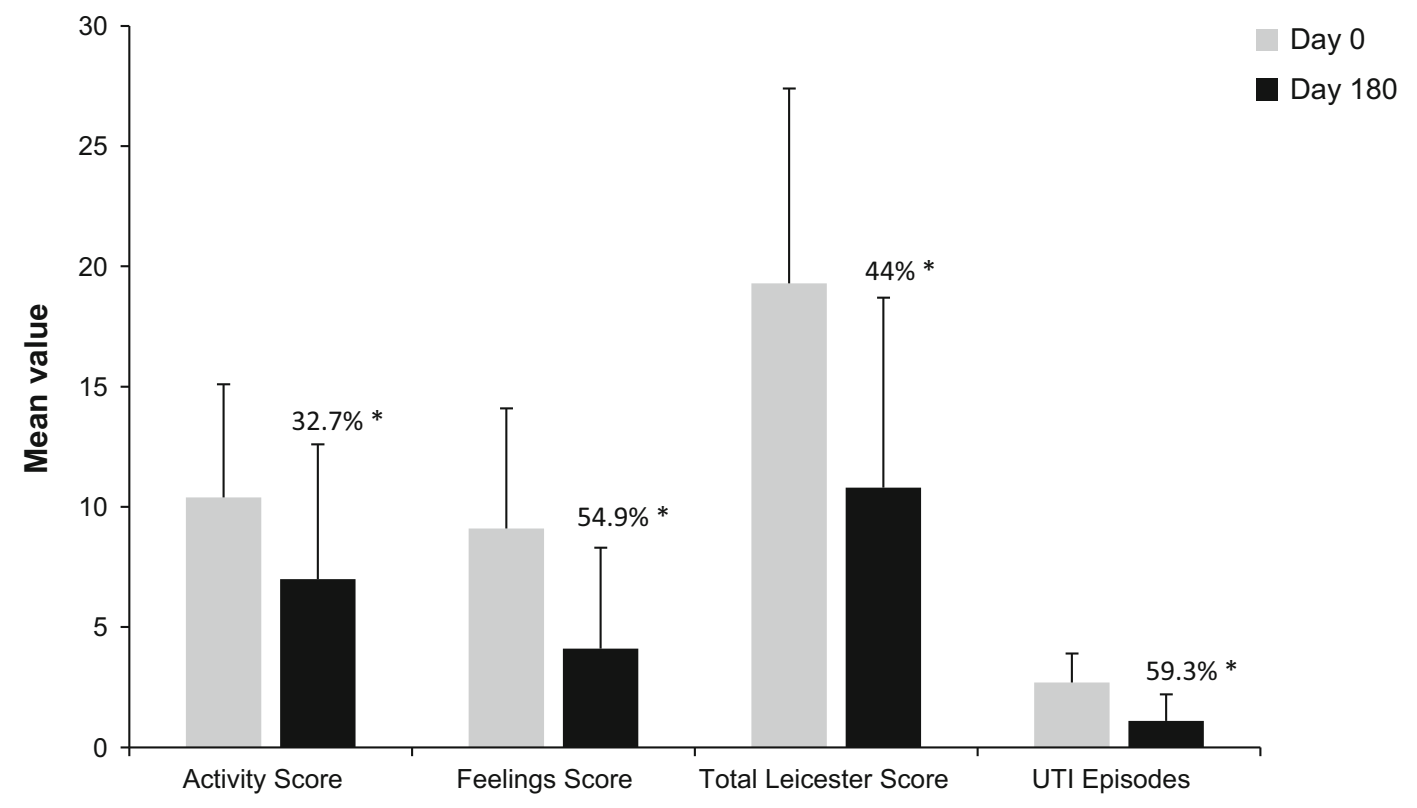

Fig. 4 Reduction of Leicester scores and number of UTIs between Day 0 and Day 180-Analysis population $(N=575)$. The Leicester impact scale was divided into four categories: $0-14,15-20,21-30$, and 31-42. A score of $>14$ indicated that the patient had some degree of

the HAD and Leicester scales indicated an improvement in QoL, which was supported by the physicians' assessment of overall improvement as 'very much better' or 'much better' in $82.4 \%$ of the study patients. The comparison between the mean HAD score and the mean number of UTIs showed a statistically significant reduction in the mean number of UTIs and a correspondingly statistically significant reduction in the overall HAD score, suggestive of an improvement in patient QoL. This was similarly revealed by a comparison between the mean Leicester score and the mean number of UTIs, reinforcing the previously described study outcomes.

Most physicians selected immunoactive E. coli extract to prevent cystitis $(94.4 \%$ of the study population were treated with immunoactive non-antimicrobial prophylaxis, OM-89), rather than prophylaxis functional or social handicap, a score of $>20$ was suggestive of a major social or functional handicap, and a score $>31$ indicated major social and functional handicaps. Asterisk the percentage reduction from Day 0. All statistically significant $(P \leq 0.0001)$. UTI urinary tract infection

with antibiotics. Interestingly, this choice of prophylaxis anticipated future EAU guidelines [1]. The immunoactive E. coli extract was first mentioned in the 2008 EAU guidelines, and was later recommended in the 2010 update [1]. In the same update, a long-term, doubleblind, placebo-controlled study [13] was referred to, which evaluated 453 patients who received either immunotherapeutic E. coli (OM-89) or matching placebo. The results showed a highly significant reduction (34\%, $P=0.001$ ) in UTI recurrences in the active treatment group compared with placebo. The current EAU guidelines [7] recommend using antimicrobial prophylaxis only when non-antimicrobial alternatives have failed. Among the available strategies, OM-89 is the most studied and has the highest level (1a) of clinical evidence, with a Grade B recommendation. 
The study's limitations are its open design and lack of control group, which introduces bias due to unblinded self-assessment. Despite its observational design, this study was a unique evaluation of the impact of rUTIs on patient QoL due to its large sample size and international multi-center design. As highlighted by Bermingham and Ashe [30], there is a paucity of data on the impact of rUTIs on QoL in the literature. This study has clearly highlighted the fact that rUTIs have a negative impact on patient QoL, shown by the prevalence of anxious-depressive symptoms and socio-functional limitations in the study population, which is consistent with results reported previously in smaller populations [18, 25]. A clear improvement in QoL provided by effective prophylaxis, such as OM-89, has been revealed by this investigation.

In conclusion, this study highlights the need for further randomized double-blind controlled trials to confirm that non-antimicrobial prophylaxis (OM-89) improves rUTI management and patient QoL.

\section{CONCLUSION}

Our results showed that rUTI had a negative impact on patients' QoL and that effective alternative prophylaxis significantly improved their QoL.

\section{ACKNOWLEDGMENTS}

We would like to thank the patients and researchers who participated in the study. This research and the associated article processing charges were supported by OM Pharma/Vifor Pharma (Geneva, Switzerland), a company of the Galenica group. All named authors meet the ICMJE criteria for authorship for this manuscript, take responsibility for the integrity of the work as a whole, and have given final approval for the version to be published.

Conflict of interest. Stefania Ballarini is OM Pharma/Vifor Pharma' employee. T. Mascarenhas, M. Zahran, E. Quimper, J. Choucair and C. Iselin were investigators in this study sponsored by OM Pharma/Vifor Pharma. Julien Renard has no conflicts of interest to declare.

Compliance with ethics guidelines. All the study procedures conformed with the ethical standards of the responsible committees on human experimentation (institutional and national) and with the 1975 Declaration of Helsinki, as revised in 2000 and 2008. Springer's policy on Informed Consent has been followed.

Open Access. This article is distributed under the terms of the Creative Commons Attribution Noncommercial License which permits any noncommercial use, distribution, and reproduction in any medium, provided the original author(s) and the source are credited.

\section{REFERENCES}

1. Grabe M, Bjerklund-Johansen TE, Botto $H$, et al. European Association of Urology guidelines on urological infections. 2010. http://www.uroweb. org. Accessed April 1, 2010.

2. McCue JD. UTIs in at-risk patients: are they 'complicated'? Infect Med. 1999;16:533-40.

3. Foxman B, Brown P. Epidemiology of urinary tract infections: transmission and risk factors, incidence, and costs. Infect Dis Clin North Am. 2003;17:227-41.

4. Foxman B. Epidemiology of urinary tract infections: incidence, morbidity, and economic costs. Am J Med. 2002;113:5S-13S. 
5. Guay DR. Contemporary management of uncomplicated urinary tract infections. Drugs. 2008;68:1169-205.

6. Gupta K, Hooton TM, Naber KG, et al. International clinical practice guidelines for the treatment of acute uncomplicated cystitis and pyelonephritis in women: a 2010 update by the Infectious Diseases Society of America and the European Society for Microbiology and Infectious Diseases. Clin Infect Dis. 2011;52:e103-20.

7. Grabe M, Bjerklund-Johansen TE, Botto $\mathrm{H}$, et al. European Association of Urology guidelines on urological infections 2014 (limited update of 2013). http://www.uroweb.org. Accessed April 1, 2014.

8. Mulvey MA, Schilling JD, Hultgren SJ. Establishment of a persistent Escherichia coli reservoir during the acute phase of a bladder infection. Infect Immun. 2001;69:4572-9.

9. Epp A, Larochelle A, Lovatsis D, et al. Recurrent urinary tract infection. J Obstet Gynaecol Can. 2010;32:1082-101.

10. Chiavaroli C, Moore A. An hypothesis to link the opposing immunological effects induced by bacterial lysate OM-89 in urinary tract infection and rheumatoid arthritis. Biodrugs. 2006;20:141-9.

11. Schulman CC, Corbusier A, Michiels H, Taenzer HJ. Oral immunotherapy of recurrent urinary tract infections: a double-blind placebo-controlled multicenter study. J Urol. 1993;150:917-21.

12. Magasi P, Panovics J, Illes A, Nagy M. Uro-Vaxom and the management of recurrent urinary tract infection in adults: a randomized multicenter double-blind trial. Eur Urol. 1994;26:137-40.

13. Bauer HW, Alloussi S, Egger G, Blümlein HM, Cozma G, Schulman CC. A long-term, multicenter, double-blind study of an Escherichia coli extract (OM-89) in female patients with recurrent urinary tract infections. Eur Urol. 2005;47:542-8.

14. Bauer HW, Rahlfs VW, Lauener PA, Blessmann GS. Prevention of recurrent urinary tract infections with immuno-active $E$. coli fractions: a metaanalysis of five placebo-controlled double-blind studies. Int J Antimicrob Agents. 2002;19:451-6.

15. Naber KG, Cho YH, Matsumoto T, Schaeffer AJ. Immunoactive prophylaxis of recurrent urinary tract infections: a meta-analysis. Int J Antimicrob Agents. 2009;33:111-9.

16. Reid G, Bruce AW. Urogenital infections in women: can probiotics help? Postgrad Med J. 2003;79:428-32.
17. Lowe NK, Ryan-Wenger NA. Military women's risk factors for and symptoms of genitourinary infections during deployment. Mil Med. 2003;168:569-74.

18. Ellis AK, Verma S. Quality of life in women with urinary tract infections: is benign disease a misnomer? J Am Board Fam Pract. 2000;13:392-7.

19. De Moura JL. Urinary infection in the adult. Acta Med Port. 1999;12:57-62.

20. Schleupner CJ. Urinary tract infections. Postgrad Med. 1997;101:231-7.

21. Bula CJ, Ghilardi G, Wietlisbach V, Petignat C, Francioli $P$. Infections and functional impairment in nursing home residents: a reciprocal relationship. J Am Geriatr Soc. 2004;52:700-6.

22. Van Oyen H, Van Oyen P. Urinary incontinence in Belgium; prevalence, correlates and psychosocial consequences. Acta Clin Belg. 2002;57:207-18.

23. Gonder MJ. Urologic considerations in the elderly. Prim Care. 1982;9:71-5.

24. Woo BK, Daly JW, Allen EC, Jeste DV, Sewell DD. Unrecognized medical disorders in older psychiatric inpatients in a senior behavioral health unit in a university hospital. J Geriatr Psychiatry Neurol. 2003;16:121-5.

25. Ernst EJ, Ernst ME, Hoehns JD, Bergus GR. Women's quality of life is decreased by acute cystitis and antibiotic adverse effects associated with treatment. Health Qual Life Outcomes. 2005;3:45.

26. Zigmond AS, Snaith RP. The hospital anxiety and depression scale. Acta Psychiatr Scand. 1983;67:361-70.

27. Shaw C, Matthews RJ, Perry SI, et al. Validity and reliability of a questionnaire to measure the impact of lower urinary tract symptoms on quality of life: the Leicester Impact Scale. Neurourol Urodyn. 2004;23:229-36.

28. Carr AJ, Gibson B, Robinson PG. Measuring quality of life: Is quality of life determined by expectations or experience? BMJ. 2001;322:1240-3.

29. Platt FW, Keating KN. Differences in physician and patient perceptions of uncomplicated UTI symptom severity: understanding the communication gap. Int $\mathrm{J}$ Clin Pract. 2007;61:303-8.

30. Bermingham SL, Ashe JF. Systematic review of the impact of urinary tract infections on health-related quality of life. BJU Int. 2012;110:E830-6. 